\title{
Axonal quantification of the white matter association fasciculi in cerebral hemispheres of cow (Bos taurus), pig (Sus scrofa domesticus) and rabbit (Oryctolagus cuniculus)
}

\author{
M. Guerrero ${ }^{1,2}$, I. Romero ${ }^{2,3}$, C. Sandoval4, 5, A. Gaibor-Pazmiño ${ }^{1}$, A. Noroña ${ }^{1}$, \\ V. Zurita' ${ }^{1}$, M. del Sol' ${ }^{2,6}$, N.E. Ottone ${ }^{2,6,7}$ \\ 'Laboratory of Anatomy, Faculty of Medical Sciences, Universidad Central del Ecuador, Quito, Ecuador \\ ${ }^{2}$ Doctoral Programme in Morphological Sciences, Faculty of Medicine, Universidad de La Frontera, Temuco, Chile \\ ${ }^{3}$ Faculty of Health Sciences, Department of Diagnostic Processes and Evaluation, School of Nutrition, Universidad \\ Católica de Temuco, Temuco, Chile \\ ${ }^{4}$ Escuela de Tecnología Médica, Facultad de Salud, Universidad Santo Tomás, Los Carreras, Osorno, Chile \\ ${ }^{5}$ Departamento de Ciencias Preclínicas, Facultad de Medicina, Universidad de La Frontera, Temuco, Chile \\ ${ }^{6}$ Centre of Excellence in Morphological and Surgical Studies (CEMyQ), Universidad de La Frontera, Temuco, Chile \\ 'Laboratory of Plastination and Anatomical Techniques, Research Centre in Dental Sciences (CICO), Faculty of Dentistry, \\ Universidad de La Frontera, Temuco, Chile
}

[Received: 22 July 2021; Accepted: 23 August 2021; Early publication date: 5 November 2021]

Background: Cerebral white matter consists mainly of axons surrounded by myelin sheaths, which are grouped to form association, commissural, and projection fasciculi. The aim of our work was to quantify and compare under the microscope the axons of the white matter association fasciculi in the cerebral hemispheres of cow (Bos taurus), pig (Sus scrofa domesticus) and rabbit (Oryctolagus cuniculus) indirectly by identification of their myelin sheaths.

Materials and methods: The samples were taken from 30 cerebral hemispheres: 10 cow, 10 pig and 10 rabbit (15 right and 15 left). They were obtained following a protocol based on the Talairach-Tournoux coordinate system for human and primate brains. The slides were stained with Luxol Fast Blue, observed by optical microscopy, and photographed at 600×. Samples were also prepared for observation in scanning transmission electron microscopy with osmium tetroxide. The myelin sheaths/axons were counted with the ImageJ software.

Results: Statistically significant differences in the number of myelin sheaths per $410 \mu \mathrm{m}^{2}$ were found in the inferior and superior longitudinal fasciculi between the left and right hemispheres of cows, with predominance of the right hemisphere; and in the inferior occipitofrontal fasciculus of the rabbit with predominance of the left hemisphere.

Conclusions: The use of animal models for experiments in the cerebral fasciculi, especially pig, could give us a greater understanding of the behaviour of demyelinating and neurodegenerative diseases in humans. (Folia Morphol 2022; 81, 4: 874-883)

Key words: axons, cerebral fasciculi, histological analysis, myelin sheaths, white matter

Address for correspondence: Dr. N.E. Ottone, Laboratory of Plastination and Anatomical Techniques, Research Centre in Dental Sciences (CICO), Dental School, Universidad de La Frontera, Temuco, Chile, e-mail: nicolas.ottone@ufrontera.cl

This article is available in open access under Creative Common Attribution-Non-Commercial-No Derivatives 4.0 International (CC BY-NC-ND 4.0) license, allowing to download articles and share them with others as long as they credit the authors and the publisher, but without permission to change them in any way or use them commercially. 


\section{INTRODUCTION}

Oligodendrocytes (OL) are one type of glial cells that provide support to the central nervous system (CNS), mainly by the formation of the myelin sheath. They extend into high numbers of branches and sub-branches expanding into sheets of myelin membranes that wrap around multiple neural axons. This myelin sheath facilitates the rapid saltatory conduction and insulation of the nerve cells [14]. It also promotes neuronal and axonal survival by secreting different types of neurotrophic factors [2, 15, 21].

Association fasciculi relate areas of the same hemisphere; the most representative are U-fasciculi, between neighbouring gyri; superior longitudinal fasciculi (SLF), inferior longitudinal fasciculi (ILF), inferior occipitofrontal fasciculi (IOFF), and uncinate fasciculi (UF), on the dorsolateral face; and cingulate fasciculi (CF) on the medial face. Commissural fasciculi relate the two hemispheres; the most important is the corpus callosum. Projection fasciculi relate the cerebral cortex with the basal ganglia; they include the internal capsule, corona radiata and optic radiations [18].

These fasciculi can be studied in vivo by functional magnetic resonance imaging, by measuring the anisotropy of the diffusion of water molecules through the axons (diffusion tension imaging [DTI]) in the fasciculi or cerebral tracts (tractography) [19].

In recent years, tractography using DTI has renewed interest in further study of white matter, as it provides a great opportunity to study its architecture. Nevertheless, this imaging technique presents two difficulties: the first is that prediction of the diffusion of water molecules follows a Gaussian distribution, when this is not really the case; the second is that the mathematical model cannot explain or adequately represent the crossing of two or more groups of fibres, interpreting them as low anisotropy. These factors reduce the reliability of the observation $[5,8]$.

Another way of studying the cerebral fasciculi is in post-mortem samples by dissection of tracts after preparation by Klingler's method [11, 24, 28, 31]. Microscopic studies can be carried out in samples of this type, in which the number of axons per fibre can be quantified; and ultramicroscopic studies to analyse axonal ultrastructure. The protocols for the conservation of samples for studies of this type allow immunohistochemical and stereological research to evaluate degenerative neuropathological mechanisms in which the axons and fasciculi are involved, such as cognitive deterioration, Parkinson's disease,
Alzheimer's disease, multiple sclerosis, and others [17]. Knowledge of adult neural stem cells both in vitro and in vivo proves to be directly pertinent to understand the capacity of the brain for self-repair, as well as yielding novel insights into brain disease research and treatment [1].

In these circumstances it is necessary to use animal models to obtain deeper, more detailed knowledge of their brains, and develop the skills to work on different segments of the brain. This will allow protocols to be established which can subsequently be applied successfully in human beings $[10,13]$. The object of this work was to quantify and compare under the microscope the axons of the white matter association fasciculi in the cerebral hemispheres of cow (Bos taurus), pig (Sus scrofa domesticus) and rabbit (Oryctolagus cuniculus) models indirectly by identification of their myelin sheaths.

\section{MATERIALS AND METHODS}

The samples used in the present work were taken from 30 cerebral hemispheres: $10 \mathrm{cow}, 10 \mathrm{pig}$ and 10 rabbit (15 right and 15 left). The complete heads of the cow and pig specimens were acquired directly from slaughterhouses, while the rabbit heads were obtained from the Animal Care and Facilitics of Universidad de La Frontera, Temuco, Chile. The approximate age of the specimens at death was 18 to 24 months for the cows, 5 to 6 months for the pigs and 2 to 3 months for rabbits. The study was approved by the Ethics Committee of Universidad de La Frontera, Temuco, Chile. The brains were extracted, and the cuts fixed following the protocol described in Figure 1.

The cuts of the samples were standardised following a protocol based on the Talairach and Tournoux [30] coordinate system for human and primate brains, adapted for cow, pig, and rabbit brains (Fig. 2).

In each cut prepared, the area of interest was selected according to the fasciculus; three-dimensional (3D) location used the coronal, sagittal and horizontal planes of the coordinate system (Fig. 3). The cuts were placed in paraffin (Sigma-Aldrich Co., St. Louis, MO, USA) following the protocol specified in Figure 4.

Five slides were obtained by microtomy from each sample block, each $8 \mu \mathrm{m}$ thick, at $20 \mu \mathrm{m}$ intervals. The samples were cut using a rotatory microtome (Leica RM2255). The slides were stained with Luxol Fast Blue (Merck, Darmstadt, Germany), following the protocol detailed in Figure 5. 


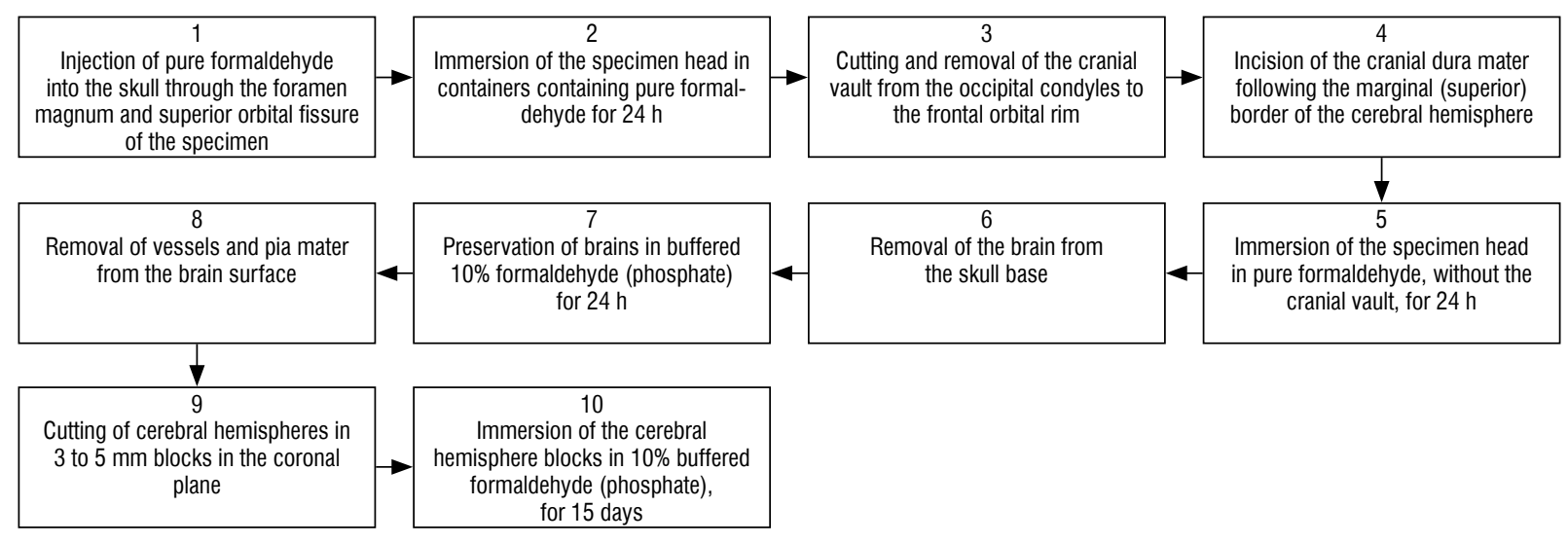

Figure 1. Protocol used to fix the cerebral hemispheres of cow, pig (1-6), and preparation of the sample for histological analysis (7-10).

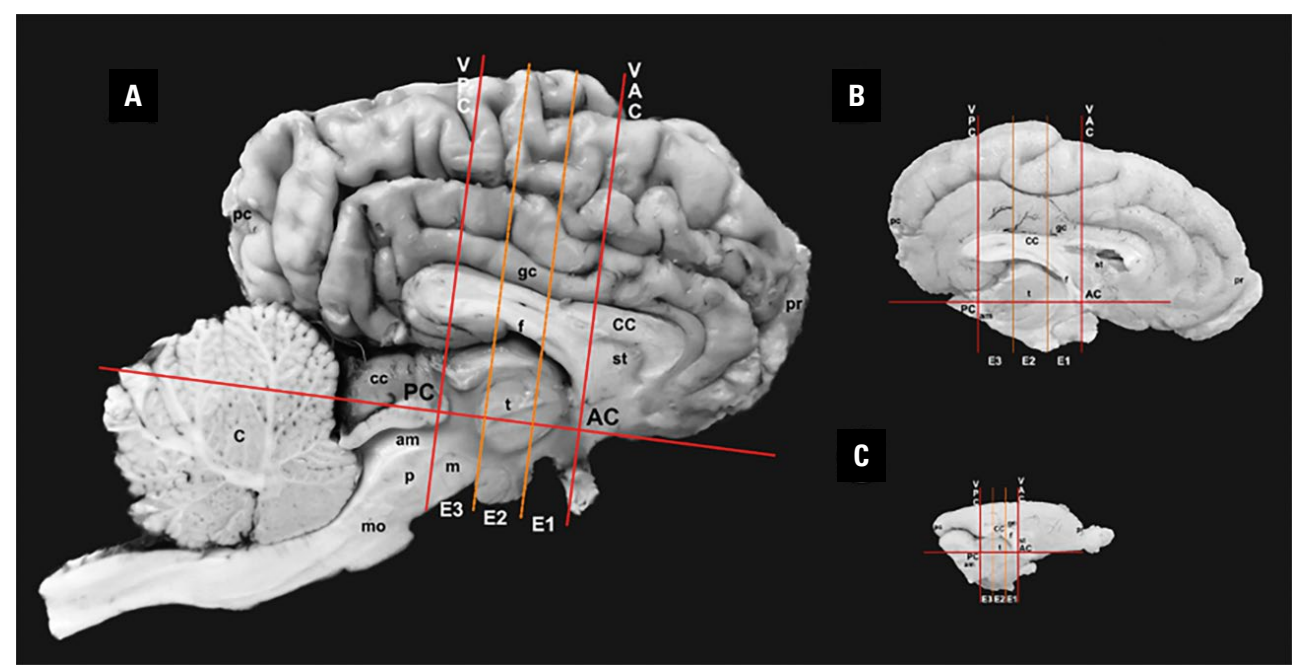

Figure 2. Talairach and Tournoux [30] standardised coordinate system for humans and primates, modified for the histological study of the association fasciculi of cow (A), pig (B) and rabbit (C) brains. The AC point (Rostral Commissure) is located on the lower border of the commissure. The PC point (Caudal Commissure) is located on the upper edge of the commissure. When these points join, the AC-PC line is formed; perpendicular to it, the vertical lines VAC and VPC are drawn and between them is the space $\mathrm{E}$ (coronal sections) which has been divided into three thirds. The parallel vertical lines are the cut lines for histological samples of the association fasciculi. Block E3 corresponds to the area of histological interest of the cingulate fasciculus, the superior longitudinal fasciculus, and the inferior longitudinal fasciculus; block E2 corresponds to the area of histological interest of the inferior occipitofrontal fasciculus and block E1 corresponds to the area of histological interest of the uncinate fasciculus.

The slides were examined in an Olympus BX53F microscope (Olympus, Tokyo, Japan); photographs of the area of interest were taken at $600 \times$ magnification with the built-in DP21 camera (Olympus, Tokyo, Japan).

Samples were also prepared of the right and left inferior occipitofrontal fasciculi of a pig's brain, using glutaraldehyde $2.5 \%$ in phosphate buffer as the primary fixer for 2 weeks (Merck, Darmstadt, Germany), and post-fixing with osmium tetroxide buffered at 2\% (Merck, Darmstadt, Germany), dehydrated with acetone (Merck, Darmstadt, Germany) in increasing concentrations from $30 \%$ to $100 \%$, interspersed with staining by uranyl acetate (Merck, Darmstadt, Ger- many). The samples were set in araldite resin (Merck, Darmstadt, Germany), also in increasing concentrations from $50 \%$ mixture with acetone to $100 \%$ and polymerised in an oven at $60^{\circ} \mathrm{C}$ for 2 days. Cuts of $150 \mathrm{~nm}$ were prepared, using 300 mesh copper grids. The images were processed by scanning transmission electron microscopy (STEM) with a high vacuum $\mathrm{Hi}$ tachi SU3500 electron microscope set for detection (Hitachi, Tokyo, Japan), with a working length of $5.7 \mathrm{~mm} \times 7.00 \mathrm{k}$.

The myelin sheaths/axons of the different fasciculi were counted using the ImageJ software (National Institute of Health), with the photographs calibrated in $\mu \mathrm{m}$. A field was selected from the most central part 


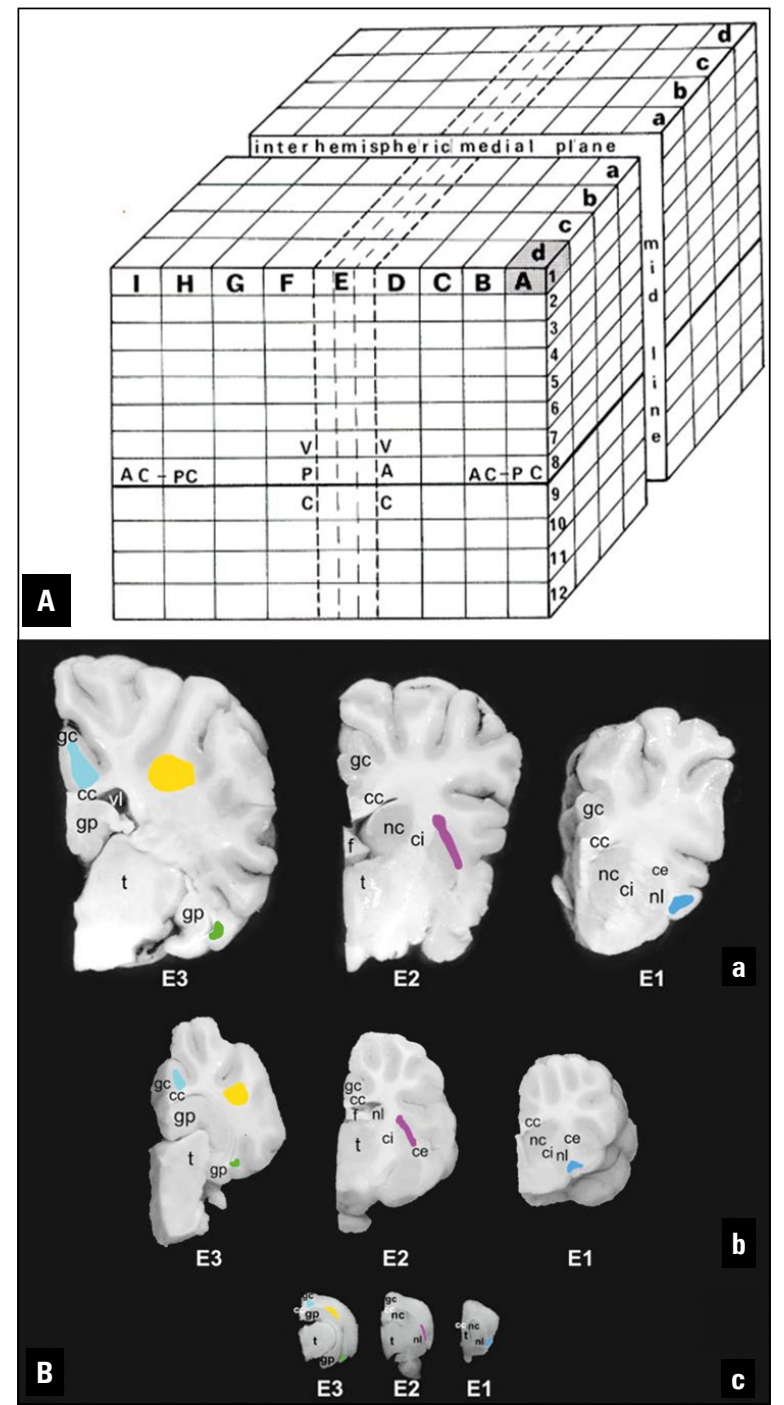

Figure 3. A. The principal planes of Talairach and Tournoux [30] can be divided by nine coronal planes $(A-I)$, by four sagittal planes (a-d) and by twelve horizontal planes (1-12). Regardless of the individual hemispherical sizes, each hemisphere will consist of a total of $9 \times 12 \times 4=432$ voxels. The relative voxel sizes of $A-D$, $\mathrm{F}-\mathrm{I}, \mathrm{a}-\mathrm{d}$ and $1-12$ are made at your convenience, respecting the proportions: $1 / 4$ rostral and caudal to $E$ in the coronal plane; $1 / 4$ medially a-d in the sagittal plane; $1 / 8$ towards the dorsal of the AC-PC line in the horizontal plane; $1 / 4$ ventrally of the AC-PC line in the horizontal plane; B. According to the Talairach and Tournoux [30] standardised coordinate system for the brain, the area of histological interest, for the sections of the association fasciculi, for this study would be found at the following coordinates: Light blue: Cingulate fasciculus: in the coronal E3 plane; in the sagittal plane 5-6; horizontally a. Red: Inferior occipitofrontal fasciculus: in the coronal plane E3; in the sagittal plane 6-7; in horizontal plane b-c. Green: Inferior longitudinal fasciculus: in the E3 coronal plane; in the sagittal plane 9-10; in horizontal plane b-c. Yellow: Superior longitudinal fasciculus: in the E2 coronal plane; in the sagittal plane 8-9; horizontal plane $a-b$. Blue: Uncinate fasciculus: in the E1 coronal plane; in the sagittal plane 9-10; in horizontal plane b-c; cc - corpus callosum; gp - gyrus parahippocampalis; gc cingulum; st - septum telencephalic; $f$ - fornix; $t$ - thalamus; nc - nucleus caudatus; ci - internal capsule; ce - outer capsule; $\mathrm{nl}$ - nucleus lentiformis; $\mathrm{vl}$ - ventriculus lateralis. of the fasciculus from each of the five slides per fasciculus; a $5 \times 4$ cell grid ( 20 cells in total) was placed over the field and five randomly selected cells were counted. The total number of fibres per fasciculus field was calculated from the known total area (field), the mean number of myelin sheaths/axons counted and the area of each cell.

These data were entered in an excel matrix and processed statistically using the SPSS $22^{\circledR}$ statistics software (IBM SPSS Statistics, IBM Corp., Armonk, NY, USA). The means, standard deviation, variance, minimum and maximum values of the data were obtained, and the p-value of the Shapiro-Wilk test was calculated to demonstrate the normality of data distribution. Statistical analysis was carried out using Student's t-test to demonstrate the difference in the number of fibres in the fasciculi between brain hemispheres of each species studied. Error bar graphs were created to show the mean with $95 \%$ confidence interval. $P<0.05$ was defined as the alpha error.

\section{RESULTS}

In the histological cuts observed through a light microscope with objective lens of $60 \times$ and ocular lens of $10 x$, the intense blue myelin sheaths can be seen surrounding the pale blue axons (Fig. 6A). Meanwhile, with the STEM, the black myelin sheaths can be observed in more detail, surrounding the grey axons (Fig. 6B).

The histological cuts of the fasciculi of the dorsolateral and medial faces of the hemispheres of the cow brain presented slight variations in the distribution of the fibres in each hemisphere. While there are no significant differences between the pig's left and right hemispheres fasciculi; differences between the inferior occipitofrontal fasciculus of the rabbit's left and right hemispheres was found ( $p=0.020$ ) (Fig. 7). In addition, the $95 \%$ confidence intervals of the cerebral fasciculi between hemispheres of the three species are shown (Fig. 8). The descriptive statistics values and the $p$ values of the fasciculi in the cow brain hemispheres are shown in Table 1. Student's t-test showed that there is a statistically significant difference between the inferior longitudinal fasciculus of the cow's left and right hemispheres ( $p=$ $=0.003$ ): the mean number of myelin sheaths found in the horizontal cut of the right hemisphere was larger than the number found in the left hemisphere. A statistically significant difference was also found in the superior longitudinal fasciculus between the 


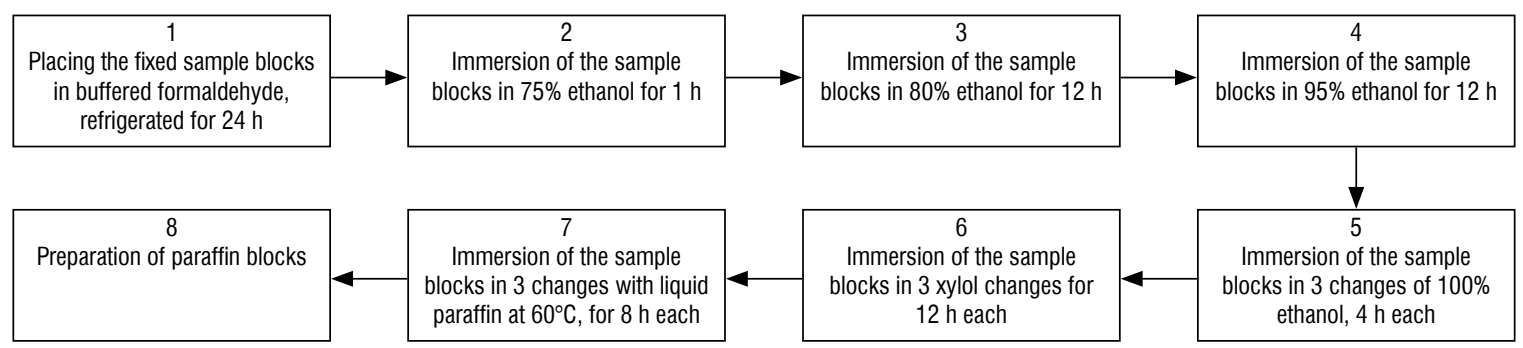

Figure 4. Paraffinisation protocol for brain section samples.

Deparaffinisation and rehydration

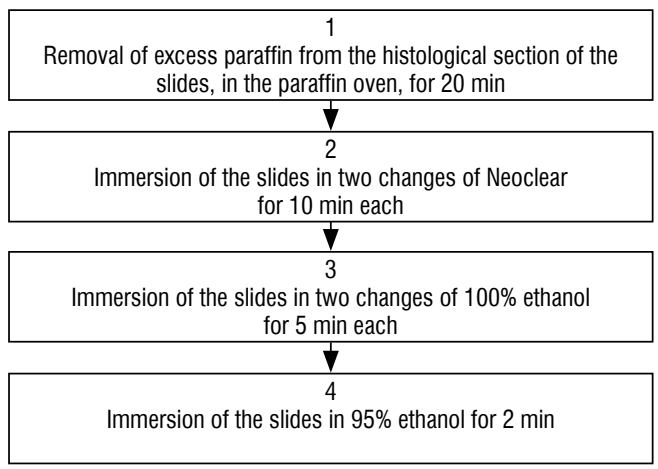

Staining and dehydration

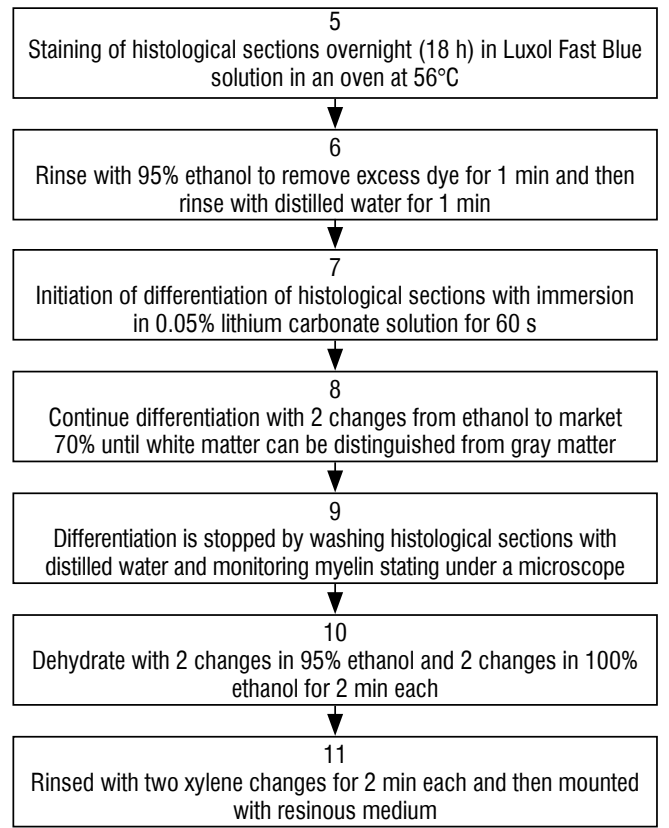

Figure 5. Protocol for deparaffinisation, staining and hydration of the histological sections. Luxol Fast Blue technique was used for staining.
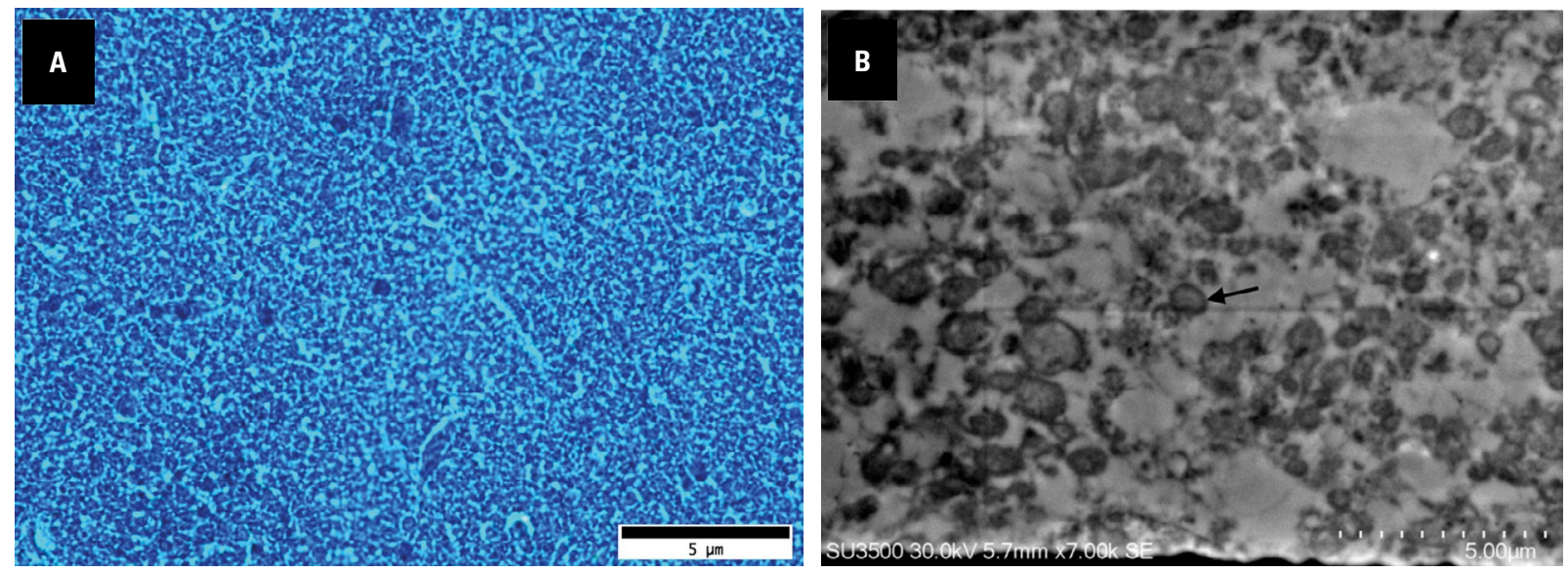

Figure 6. A. Photomicrograph of a histological section at $600 \times($ objective lens $60 \times)$ of the area of interest of the pig's $z$ cingulate fasciculus where axons (light blue colour) can be seen cut transversely with their myelin sheaths (dark blue colour) forming small circles your surroundings. Staining: Luxol Fast Blue; B. Ultramicrophotography of a histological section processed in scanning transmission electron microscopy (7k), of the area of interest of the pig's cingulate fasciculus where the transversely cut myelin sheaths with black coloration (black arrowhead) can be seen forming circles around the fibre nervous (grey colour). Staining: Osmium Tetroxide. 


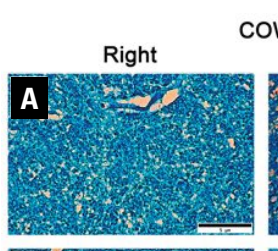

cow
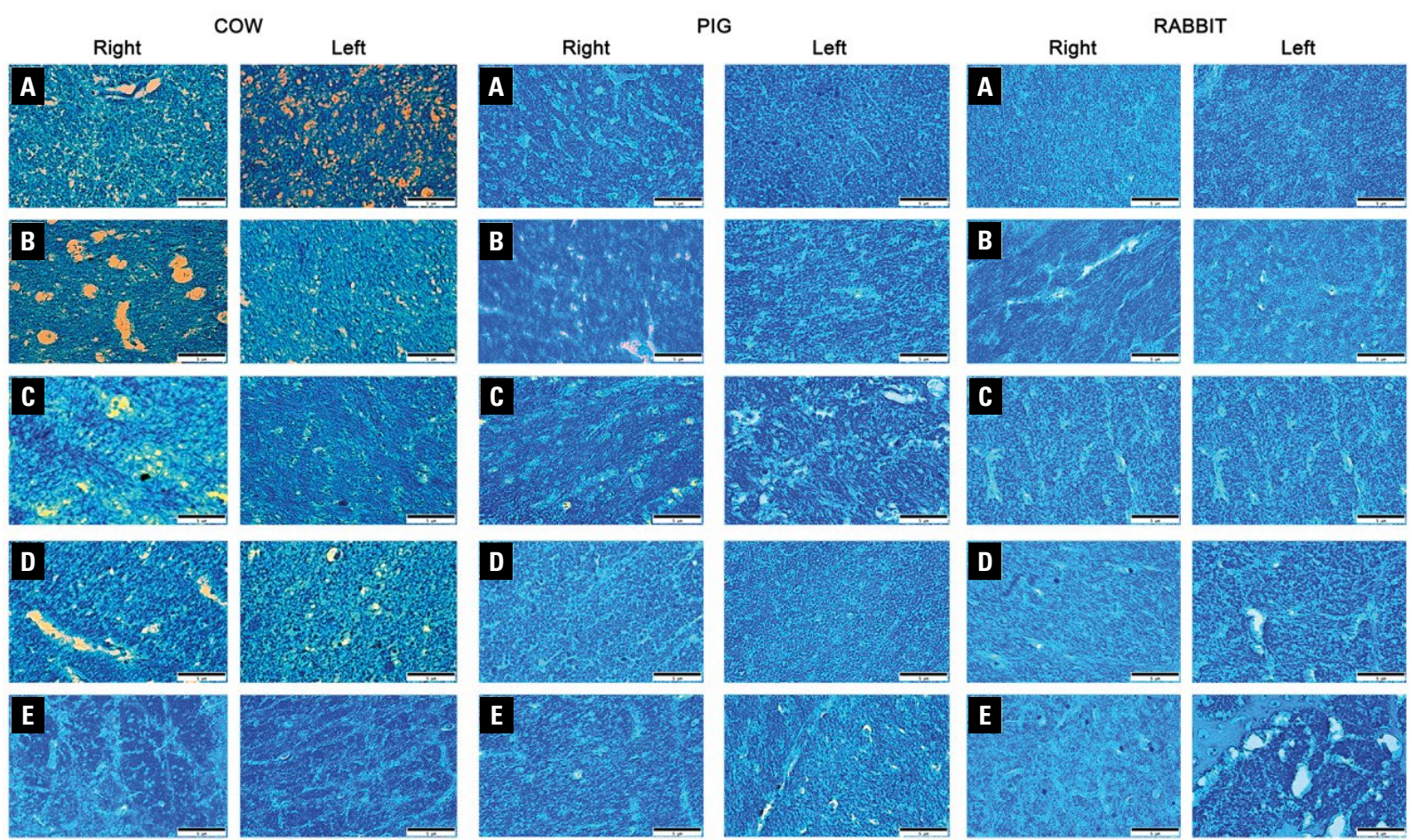

Figure 7. Cow. Microphotographs of histological sections at $600 \times(60 \times$ objective lens) of cow cerebral hemispheres where the myelin sheaths (of an intense blue colour) can be seen surrounding the myelinated axons (of light blue colour) of the fasciculi. $410 \mu \mathrm{m}^{2}$ area of interest; A. Cingulate fasciculus; B. Inferior occipitofrontal fasciculus; C. Inferior longitudinal fasciculus; D. Superior longitudinal fasciculus;

E. Uncinate fasciculus. Staining: Luxol Fast Blue. Pig. Microphotographs of histological sections at $600 \times(60 \times$ objective lens) of the cerebral hemispheres of pigs, where the myelin sheaths (of an intense blue colour) can be seen surrounding the myelinated axons (of light blue colour) of the fasciculi. $410 \mu \mathrm{m}^{2}$ area of interest; A. Cingulate fasciculus; B. Inferior occipitofrontal fasciculus; C. Inferior longitudinal fasciculus; D. Superior longitudinal fasciculus; E. Uncinate fasciculus. Staining: Luxol Fast Blue. Rabbit. Microphotographs of histological sections at $600 \times(60 \times$ objective lens) of rabbit cerebral hemispheres where the myelin sheaths (of an intense blue colour) can be seen surrounding the myelinated axons (of light blue colour) of the fasciculi. $410 \mu \mathrm{m}^{2}$ area of interest; A. Cingulate fasciculus; B. Inferior occipitofrontal fasciculus; C. Inferior longitudinal fasciculus; D. Superior longitudinal fasciculus; E. Uncinate fasciculus. Staining: Luxol Fast Blue.

hemispheres $(p=0.008)$ : the mean number of myelin sheaths found in the horizontal cut of the right hemisphere was larger than found in the left hemisphere (Tables 2 and 3).

\section{DISCUSSION}

The present study started from the premises that it is possible to identify, isolate and relate the white matter fasciculi of the dorsolateral and medial faces of the brain hemispheres in the experimental animals used, namely: CF, IOFF, ILF, SLF, UF, using tract preparation and dissection techniques to recognize their form, distribution, size, and width [4, 22, 24, 27].

One of the problems that had to be solved to obtain standardised histological samples of optimum quality and representativeness was finding the right site to make the cuts of each fasciculus, considering its distribution and its inter-relation and crossover with other fasciculi; reference points were therefore established from which the location and size could be standardised. Various standardized procedures with stable reference points were found in the scientific literature to facilitate access to brain structures in both animals and humans, e.g., stereo-encephalotomy [29], the first stereotaxic atlas of the human brain; radiological, nuclear magnetic resonance, digital and 3D atlases; and the Co-Planar Stereotaxic Atlas of the Human Brain by Talairach and Tournoux [30].

Atlases of the brains of animal models were also reviewed, e.g., rat [23], rabbit [20,26], pig [3, 9, 22]. A common element in most of stereotaxic atlases is the selection of two reference points which are very stable in any mammal brain, whether gyrencephalic or lissencephaly; these are known as the anterior and posterior commissures in humans, or the rostral and caudal commissures in animals. They are joined by a referential line, called the intercommissural line, and this line is crossed by perpendicular lines which 

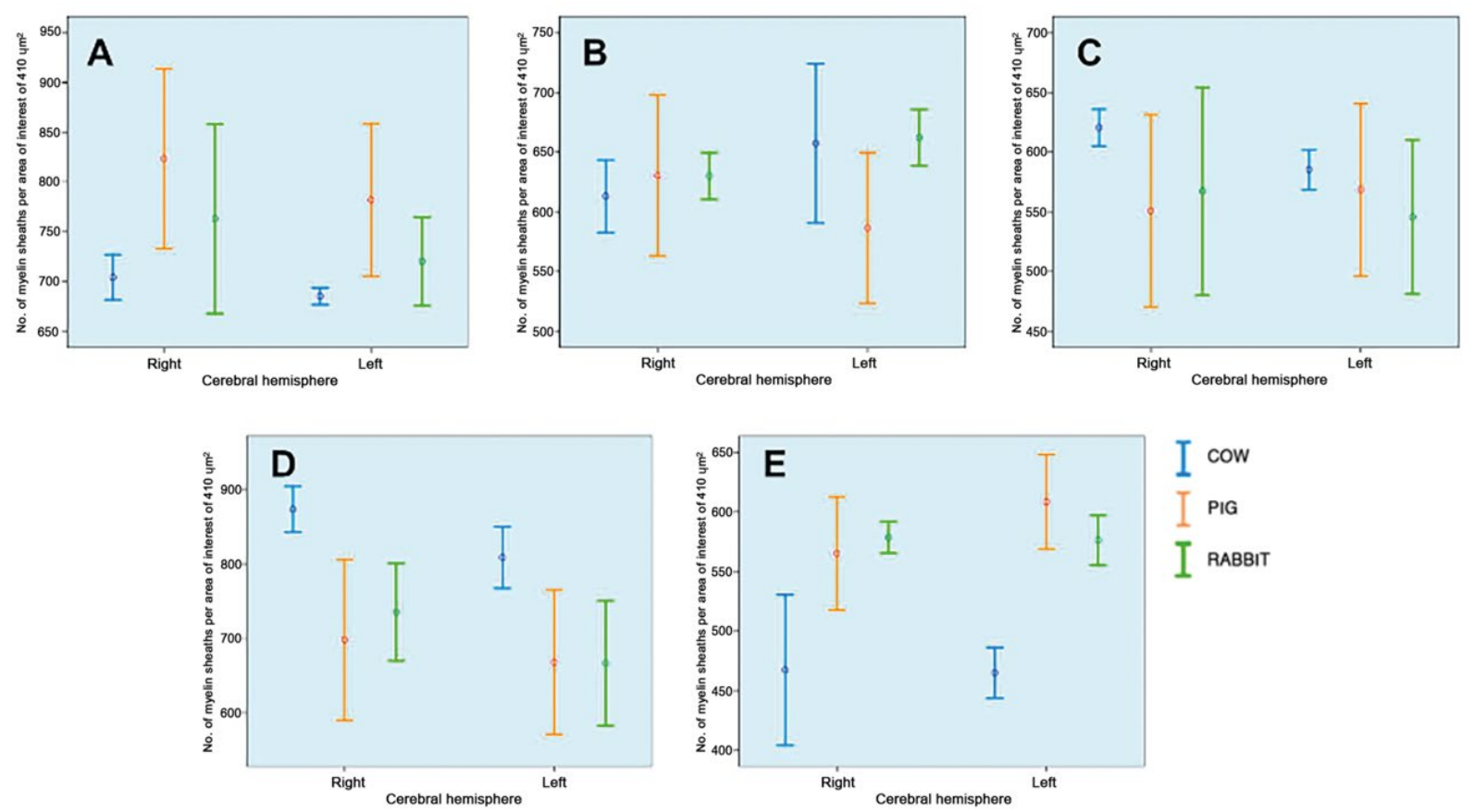

Figure 8. Comparison of the mean and $95 \%$ confidence interval of cingulate fasciculi (CF) (A), inferior occipitofrontal fasciculi (IOFF) (B), inferior longitudinal fasciculi (ILF) (C), superior longitudinal fasciculi (SLF) (D) and uncinate fasciculi (UF) (E), between the cerebral hemispheres of cow, pig, and rabbit. Statistically significant differences can be seen in the mean values of ILF and SLF myelin sheaths in cattle; and the mean values of the rabbit IOFF myelin sheaths.

Table 1. Statistical values of the number of myelin sheaths per $410 \mu \mathrm{m}^{2}$ field in cross sections of the association brain fasciculi of the cow

\begin{tabular}{|c|c|c|c|c|c|c|c|c|c|c|c|}
\hline \multirow[t]{2}{*}{ Fasciculus } & \multicolumn{5}{|c|}{ Right hemisphere (n = 5) } & \multicolumn{5}{|c|}{ Left hemisphere ( $n=5$ ) } & \multirow[t]{2}{*}{$\mathbf{P}$} \\
\hline & Mean \pm SD & $\mathbf{S}^{2}$ & Min & Max & S-W & Mean \pm SD & $\mathbf{S}^{2}$ & Min & Max & S-W & \\
\hline $\mathrm{CF}$ & $704 \pm 18.3$ & 334.0 & 690 & 733 & 0.152 & $685 \pm 6.8$ & 46.5 & 674 & 692 & 0.403 & 0.061 \\
\hline IOFF & $613 \pm 24.5$ & 602.0 & 592 & 640 & 0.052 & $657 \pm 53.9$ & 2902.3 & 602 & 741 & 0.575 & 0.132 \\
\hline ILF & $621 \pm 12.5$ & 155.3 & 611 & 642 & 0.081 & $585 \pm 13.5$ & 183.3 & 567 & 598 & 0.404 & 0.003 \\
\hline SLF & $874 \pm 24.9$ & 621.3 & 837 & 899 & 0.568 & $809 \pm 33.4$ & 1113.2 & 774 & 855 & 0.617 & 0.008 \\
\hline UF & $467 \pm 51.0$ & 2602.2 & 425 & 547 & 0.279 & $465 \pm 17.2$ & 295.7 & 445 & 484 & 0.506 & 0.923 \\
\hline
\end{tabular}

SD - standard deviations; Min - minimum; Max - maximum; $\mathrm{S}^{2}$ - variance; S-W - Shapiro-Wilks's test; P — probability value of the comparison of means between the cerebral hemispheres; CF — cingulate fasciculus; IOFF — inferior occipitofrontal fasciculus; ILF — inferior longitudinal fasciculus; SLF — superior longitudinal fasciculus; UF — uncinate fasciculus

Table 2. Statistical values of the number of myelin sheaths per $410 \mu \mathrm{m}^{2}$ field in cross sections of the association brain fasciculi of the pig

\begin{tabular}{|c|c|c|c|c|c|c|c|c|c|c|c|}
\hline \multirow[t]{2}{*}{ Fasciculus } & \multicolumn{5}{|c|}{ Right hemisphere ( $n=5$ ) } & \multicolumn{5}{|c|}{ Left hemisphere $(n=5)$} & \multirow[t]{2}{*}{$\mathbf{P}$} \\
\hline & Mean \pm SD & $\mathbf{S}^{2}$ & Min & Max & S-W & Mean \pm SD & $\mathbf{S}^{2}$ & Min & Max & S-W & \\
\hline $\mathrm{CF}$ & $823 \pm 73.0$ & 5331.8 & 698 & 872 & 0.041 & $782 \pm 62$ & 3844.2 & 693 & 862 & 0.95 & 0.173 \\
\hline IOFF & $630 \pm 54.4$ & 2961.3 & 551 & 698 & 0.965 & $586 \pm 50.8$ & 2585.3 & 531 & 652 & 0.656 & 0.223 \\
\hline ILF & $551 \pm 65.0$ & 4221.2 & 496 & 655 & 0.291 & $568 \pm 58.3$ & 3399.3 & 490 & 631 & 0.748 & 0.664 \\
\hline SLF & $698 \pm 87.0$ & 7568.0 & 600 & 790 & 0.346 & $668 \pm 78.3$ & 6136.5 & 584 & 751 & 0.260 & 0.582 \\
\hline UF & $565 \pm 38.3$ & 1463.7 & 521 & 611 & 0.380 & $609 \pm 32.0$ & 1023.3 & 560 & 638 & 0.384 & 0.088 \\
\hline
\end{tabular}

SD — standard deviations; Min — minimum; Max — maximum; $S^{2}$ — variance; S-W — Shapiro-Wilks's test; P — probability value of the comparison of means between the cerebral hemispheres; CF — cingulate fasciculus; IOFF — inferior occipitofrontal fasciculus; ILF — inferior longitudinal fasciculus; SLF — superior longitudinal fasciculus; UF — uncinate fasciculus 
Table 3. Statistical values of the number of myelin sheaths per $410 \mu \mathrm{m}^{2}$ field in cross sections of the association brain fasciculi of the rabbit

\begin{tabular}{|c|c|c|c|c|c|c|c|c|c|c|c|}
\hline \multirow[t]{2}{*}{ Fasciculus } & \multicolumn{5}{|c|}{ Right hemisphere ( $n=5$ ) } & \multicolumn{5}{|c|}{ Left hemisphere $(n=5)$} & \multirow[t]{2}{*}{$\mathbf{P}$} \\
\hline & Mean \pm SD & $\mathbf{S}^{2}$ & Min & Max & S-W & Mean \pm SD & $\mathbf{S}^{2}$ & Min & Max & S-W & \\
\hline CF & $763 \pm 76.8$ & 5905.5 & 688 & 883 & 0.441 & $720 \pm 35.9$ & 1286.0 & 678 & 768 & 0.922 & 0.290 \\
\hline IOFF & $630 \pm 15.7$ & 247.0 & 603 & 643 & 0.096 & $662 \pm 19.1$ & 364.2 & 642 & 684 & 0.326 & 0.020 \\
\hline ILF & $567 \pm 70.1$ & 4920.7 & 492 & 661 & 0.733 & $546 \pm 52.0$ & 2698.8 & 486 & 621 & 0.743 & 0.595 \\
\hline SLF & $735 \pm 52.8$ & 2789.3 & 684 & 795 & 0.149 & $667 \pm 67.6$ & 4574.7 & 610 & 752 & 0.114 & 0.112 \\
\hline UF & $579 \pm 10.6$ & 113.3 & 569 & 596 & 0.273 & $576 \pm 16.9$ & 284.2 & 558 & 594 & 0.258 & 0.795 \\
\hline
\end{tabular}

SD — standard deviations; Min — minimum; Max — maximum; $\mathrm{S}^{2}$ — variance; S-W — Shapiro-Wilks's test; P — probability value of the comparison of means between the cerebral hemispheres; CF — cingulate fasciculus; IOFF — inferior occipitofrontal fasciculus; ILF — inferior longitudinal fasciculus; SLF — superior longitudinal fasciculus; UF — uncinate fasciculus

also cross the commissures, known as vertical lines. These elements were basic for the construction of the Co-Planar Stereotaxic Atlas of the Human Brain of Talairach and Tournoux [30], which was the main reference used for obtaining the samples in this histological study.

Various protocols were tested for the dehydration and subsequent paraffinisation of the cuts, considering that the sample blocks were fixed in buffered formalin for 15 days and not frozen. Several techniques have been developed to view the myelinated axons which abound in the CNS, depending on the nature of the study. Techniques exist which use the colloidal or Gallyas plate but produce variable results depending on the post-mortem handling of the tissue, the ambient temperature during physical development, the quality of the reagents used and the competence of the experimenter carrying out the technique [25]. There is also the osmium tetroxide technique; this is more frequently used for electron microscopy, since in a light microscope the myelin sheaths appear black, obscuring many cytoarchitectural characteristics of the axons. Histochemical methods for hydrophilic phospholipids are better used with frozen brain sections and are more useful for confirming the presence of products of myelin degeneration [7].

Staining with Luxol Fast Blue was selected because of the lipoprotein compounds contained in the myelin, which are resistant to organic solvents; associated with phospholipids they transfer the hydrophobic properties contained in the anionic heads of the polar lipids. These are retained in the myelin, which is more hydrophilic than fat but more hydrophobic than cytoplasm or collagen, producing a better view of the myelin sheaths surrounding the axons for the purposes of linear morphometry. This allows the cerebral fasciculi to be distinguished and their limits to be seen more clearly $[7,16]$.
No works were found in the literature which describe histological analysis of the number of axons in the cerebral fasciculi; however, works were found using DTI which used probabilistic calculation of the anisotropy of water to find the direction and number of axonal fibres contained. The problem that arises here is that because it is a probability, the numbers are not exact - the more so when the fibres change direction or cross other fasciculi $[6,8,11]$.

The staining method used in the present study allowed us to count the myelin sheaths/axons in each fasciculus. It was found that in general the number of myelin sheaths/axons is symmetrical between the fasciculi of the left and right hemispheres of each species, except in the superior and inferior longitudinal fasciculi of the cow, where there was a clear predominance of the right hemisphere. This is consistent with a previous study by the authors, where a statistically significant difference was also found in the width of these fasciculi [12]. These findings would in theory corroborate the dominance of one hemisphere, in the areas of motor-auditive-visual coordination related with the superior longitudinal fasciculus; and olfactory-visual coordination related with the inferior longitudinal fasciculus.

Another aspect which merits special attention in future investigations, especially in the use of animal models for neuroscientific exploration, is that the number of myelin fibres per field explored in the cerebral fasciculi of the pig was greater than in the other animal models used. This reconfirms that the brain of this animal model bears more similarity to the human brain, which is why it has frequently been used for physio-pathological studies of Parkinson's disease, psychostimulant drugs, neurotransmitters, and anatomic-radiological correlation studies [3]. 


\section{Limitations of the study}

As this was a histological study, the main limitation is the fact that the structures studied were subjected to fixing substances which could alter the architecture of the brain structures studied. No calculations were applied to compensate any such alterations since all the structures were subjected to the same laboratory conditions.

\section{CONCLUSIONS}

The protocols proposed here, from the fixing of brain tissue to mounting and staining, the standardisation of coordinates for sample extraction, and the procedure for counting myelin sheaths/axons, will allow these elements to be identified and compared between the different species studied. Reliable results can be obtained due to the rigor of the methods used, which may be beneficial for adapting the protocols of these morphometric studies to post-mortem studies of human brains in pursuit of better understanding of the processes of degenerative diseases of the CNS like senile dementia, Alzheimer's disease, Parkinson's disease, multiple sclerosis, between others.

\section{Conflict of interest: None declared}

\section{REFERENCES}

1. Abdissa D, Hamba N, Gerbi A. Review Article on adult neurogenesis in humans. Transl Res Anat. 2020; 20: 100074, doi: 10.1016/j.tria.2020.100074.

2. Adeeb N, Deep A, Hose N, et al. Stem cell therapy for spinal cord injury: The use of oligodendrocytes and motor neurons derived from human embryonic stem cells. Transl Res Anat. 2015; 1: 17-24, doi: 10.1016/j.tria.2015.10.003.

3. Andersen F, Watanabe $H$, Bjarkam $C$, et al. Pig brain stereotaxic standard space: mapping of cerebral blood flow normative values and effect of MPTP-lesioning. Brain Res Bull. 2005; 66(1): 17-29, doi: 10.1016/j.brainresbull.2005.02.033, indexed in Pubmed: 15925140.

4. Andronikou S. White Matter Tracts. In: (ed.). See Right Through Me. An Imaging Anatomy Atlas. Springer, Amsterdam 2012.

5. Assaf Y, Pasternak O. Diffusion tensor imaging (DTI)-based white matter mapping in brain research: a review. J Mol Neurosci. 2008; 34(1): 51-61, doi: 10.1007/s12031-0070029-0, indexed in Pubmed: 18157658.

6. Bürgel $U$, Amunts $K$, Hoemke $L$, et al. White matter fiber tracts of the human brain: three-dimensional mapping at microscopic resolution, topography and intersubject variability. Neuroimage. 2006; 29(4): 1092-1105, doi: 10.1016/j.neuroimage.2005.08.040, indexed in Pubmed: 16236527.

7. Carriel V, Campos A, Alaminos M, et al. Staining methods for normal and regenerative myelin in the nervous system. Methods Mol Biol. 2017; 1560: 207-218, doi: 10.1007/978-1-4939-6788-9_15, indexed in Pubmed: 28155156.

8. Catani M, Thiebaut de Schotten M. A diffusion tensor imaging tractography atlas for virtual in vivo dissections. Cortex. 2008; 44(8): 1105-1132, doi: 10.1016/j.cortex.2008.05.004, indexed in Pubmed: 18619589.

9. Félix $B$, Léger $M E$, Albe-Fessard $D$, et al. Stereotaxic atlas of the pig brain. Brain Res Bull. 1999; 49(1-2): 1-137, doi: 10.1016/s0361-9230(99)00012-x, indexed in Pubmed: 10466025.

10. Fernández-Miranda JC, Rhoton AL, Alvarez-Linera J, et al. Three-dimensional microsurgical and tractographic anatomy of the white matter of the human brain. Neurosurgery. 2008; 62(6 Suppl 3): 989-1026; discussion 1026, doi: 10.1227/01.neu.0000333767.05328.49, indexed in Pubmed: 18695585.

11. Guerrero M, del-Sol M, Ottone N. Preparación de Hemisferios Cerebrales para Disección de Tractos. Int J Morphol. 2019; 37(2): 533-540, doi: 10.4067/s071795022019000200533.

12. Guerrero M, Veuthey C, Del Sol M, et al. Dissection of white matter association fasciculi in bovine (Bos taurus), pig (Sus scrofa domesticus) and rabbit (Oryctolagus cuniculus) brains. Anat Histol Embryol. 2020; 49(4): 550-562, doi: 10.1111/ahe.12561, indexed in Pubmed: 32281688.

13. Ikeda K, Shoin K, Mohri M, et al. Surgical indications and microsurgical anatomy of the transchoroidal fissure approach for lesions in and around the ambient cistern. Neurosurgery. 2002; 50(5): 1114-1120, doi: 10.1097/00006123-200205000-00030, indexed in Pubmed: 11950415.

14. Izrael M, Zhang $P$, Kaufman R, et al. Human oligodendrocytes derived from embryonic stem cells: Effect of noggin on phenotypic differentiation in vitro and on myelination in vivo. Mol Cell Neurosci. 2007; 34(3): 310-323, doi: 10.1016/j.mcn.2006.11.008, indexed in Pubmed: 17196394.

15. Keirstead HS, Nistor G, Bernal G, et al. Human embryonic stem cell-derived oligodendrocyte progenitor cell transplants remyelinate and restore locomotion after spinal cord injury. J Neurosci. 2005; 25(19): 4694-4705, doi: 10.1523/ JNEUROSCI.0311-05.2005, indexed in Pubmed: 15888645.

16. Kiernan J. Histochemistry of staining methods for normal and degenerating myelin in the central and peripheral nervous systems. J Histotechnol. 2013; 30(2): 87-106, doi: 10.1179/his.2007.30.2.87.

17. Liu XB, Schumann CM. Optimization of electron microscopy for human brains with long-term fixation and fixed-frozen sections. Acta Neuropathol Commun. 2014; 2: 42, doi: 10.1186/2051-5960-2-42, indexed in Pubmed: 24721148.

18. Martino J, De Lucas EM. Subcortical anatomy of the lateral association fascicles of the brain: a review. Clin Anat. 2014; 27(4): 563-569, doi: 10.1002/ca.22321, indexed in Pubmed: 24453050.

19. Melhem ER, Mori S, Mukundan G, et al. Diffusion tensor MR imaging of the brain and white matter tractography. Am J Roentgenol. 2002; 178(1): 3-16, doi: 10.2214/ ajr.178.1.1780003, indexed in Pubmed: 11756078.

20. Muñoz-Moreno E, Arbat-Plana A, Batalle D, et al. A magnetic resonance image based atlas of the rabbit brain for automat- 
ic parcellation. PLoS One. 2013; 8(7): e67418, doi: 10.1371/ journal.pone.0067418, indexed in Pubmed: 23844007.

21. Nistor Gl, Totoiu MO, Haque N, et al. Human embryonic stem cells differentiate into oligodendrocytes in high purity and myelinate after spinal cord transplantation. Glia. 2005; 49(3): 385-396, doi: 10.1002/glia.20127, indexed in Pubmed: 15538751.

22. Pascalau R, Szabo B. Fibre dissection and sectional study of the major porcine cerebral white matter tracts. Anat Histol Embryol. 2017; 46(4): 378-390, doi: 10.1111/ahe.12280, indexed in Pubmed: 28677169.

23. Paxinos J, Waltson C. The Rat Brain in Stereotaxic Coordinates. 5th ed. Elsevier Academic Press, Hoboken 2005.

24. Pérez CJC, Gallegos SSP, Garduño PP, et al. Estandarización del método Klingler y su visualización tridimensional. Rev Hosp Jua Mex. 2008; 75(2): 99-108.

25. Pistorio AL, Hendry SH, Wang X. A modified technique for high-resolution staining of myelin. J Neurosci Methods. 2006; 153(1): 135-146, doi: 10.1016/j.jneumeth.2005.10.014, indexed in Pubmed: 16310256.

26. Sawyer CH, Everett JW, Green JD. The rabbit diencephalon in stereotaxic coordinates. J Comp Neurol. 1954; 101(3): 801-824, doi: 10.1002/cne.901010307, indexed in Pubmed: 13233360 .
27. Shah A, Jhawar SS, Goel A. Analysis of the anatomy of the Papez circuit and adjoining limbic system by fiber dissection techniques. J Clin Neurosci. 2012; 19(2): 289-298, doi: 10.1016/j.jocn.2011.04.039, indexed in Pubmed: 22209397.

28. Silva SM, Andrade JP. Neuroanatomy: The added value of the Klingler method. Ann Anat. 2016; 208: 187-193, doi: 10.1016/j.aanat.2016.06.002, indexed in Pubmed: 27329122

29. Spiegel EA, Wycis HT, Freed H. Stereoencephalotomy in thalamotomy and related procedures. J Am Med Assoc. 1952; 148(6): 446-451, doi: 10.1001/ jama.1952.02930060028009, indexed in Pubmed: 14888502.

30. Talairach J, Tournoux P. Co-planar stereotaxic atlas of the human brain. 3-dimensional proportional system: an approach to cerebral imaging. Georg Thieme, New York 1998.

31. Zemmoura I, Blanchard E, Raynal PI, et al. How Klingler's dissection permits exploration of brain structural connectivity? An electron microscopy study of human white matter. Brain Struct Funct. 2016; 221(5): 2477-2486, doi: 10.1007/s00429-015-1050-7, indexed in Pubmed: 25905864 . 